\title{
TITLE: CEREBROVASCULAR EFFECTS OF RETROPERITONEAL LAPAROSCOPY IN CHILDREN
}

Cengiz Karsli MD, Mirko Dolci MD, Igor Luginbuehl MD, Walid Farhat MD, Bruno Bissonnette MD University of Toronto, Department of Anesthesia, Sick Kids, 555 University Avenue, Toronto, Ontario, M5G 1X8

INTRODUCTION: Retroperitoneal laparoscopy is a technique which is being used with increasing regularity.(1) Cerebral blood flow velocity has been shown to vary directly with $\mathrm{ETCO}_{2}$ in children undergoing intraperitoneal laparoscopy.(2) This study examines the cerebral hemodynamic effects of retroperitoneal $\mathrm{CO}_{2}$ insufflation in children.

METHODS: With REB approval and written parental consent pediatric patients undergoing laparoscopic partial or total nephrectomy were enrolled. A standardized anesthetic technique of isoflurane $1 \mathrm{MAC}$ and remifentanil $0.2 \mu \mathrm{g} / \mathrm{kg} / \mathrm{min}$ was used. Parameters measured included peak airway pressure, end-tidal $\mathrm{CO}_{2}$ and middle cerebral artery blood flow velocity (Vmca). Transcranial Doppler sonography was used to measure Vmca. Data was collected before, during and after $12 \mathrm{mmHg}$ of retroperitoneal pneumoperitoneum, at regular 1-2 minute intervals. Within group analysis was performed using repeated measures ANOVA and values at different points of time were compared using paired t-test. $P<0.05$ was considered significant.

RESULTS: Twelve participants with a mean age of $6.5 \pm 4.8 \mathrm{yr}$ and weight of $23.9 \pm$ $13.5 \mathrm{~kg}$ have been recruited to date. Both Vmca and end-tidal $\mathrm{CO}_{2}$ tended to increase progressively throughout the study period. All Vmca and end-tidal $\mathrm{CO}_{2}$ values from 3 minutes onwards were significantly higher than baseline $(\mathrm{P}<0.01)$.

DISCUSSION: Changes in cerebral blood flow velocity in children undergoing retroperitoneal laparoscopy seem to be related to the resultant effects on end-tidal $\mathrm{CO}_{2}$. This effect seems to be similar to that seen during intraperitoneal laparoscopy.(2)

\section{REFERENCES:}

1. J Uro l160:1138-41.

2. Asso cAn asth ofGB an dIrelan dabstract, 2001 